frequency of cases of theft among boys than among girls, would have been better grounded if Prof. Burt had been able to produce independent evidence to show that women are innately less acquisitive than men. One conclusion certainly emerges from this discussion, namely, the urgent need there is at present for a really scientific classification of the human instincts. The volume before us is the first of a series of three. The other two will be eagerly awaited by all students of social science and education. Morris GINSBERG.

\section{The Structure of the Earth.}

Der Aufbau der Erde. Von Dr. B. Gutenberg. Pp. viii + r68. (Berlin : Gebrüder Borntraeger, r925.) 9 gold marks.

$7 \mathrm{HE}$ work under review is a general survey of the 1 known results relating to the condition of the interior of the earth. In most cases the theories discussed are not developed in detail, but summaries of the data and the main conclusions are given. Accordingly a very large amount of material has been compressed into a small space. It may be said that the main use of the book is to facilitate reference to original investigations; in this the reviewer speaks from his own experience. Perhaps the greatest difficulty that besets geophysics is that its literature is scattered through so many periodicals. It is easy to think of eight British journals that have contained important geophysical (apart from meteorological) papers during the past ten years, but in Germany the confusion is even greater. The bibliography in this book refers to twenty papers by the author himself, in nine periodical and several non-periodical publications ; and the total number of German periodicals including geophysical papers is such that some convenient summary, with references, such as Dr. Gutenberg's book affords, is essential to any one wishing to know what work has actually been carried out. Unfortunately there are signs of insufficient verification; near the beginning of the bibliography we find "Cooker" for "Coker," without indication that the associated Adams is F. D. and not J. C. or L. H. ; and incorrect initials are then attributed several times to $L$. H. Adams. But the labour involved in compiling a I4-page bibliography in the circumstances must have been enormous, and the author deserves our gratitude, especially as he has done justice to countries and languages not his own.

The seismological parts of the book merit the greatest attention. The illustrations on pp. $7,8,25,30$, and 96 , showing typical seismograms for various epicentral distances, are a valuable feature. Accounts are given of the determination of the wave-velocities at various depths from the times of transmission, and also of Zöppritz's method of amplitudes, the actual application of which is largely due to Dr. Gutenberg. If the velocity of a compressional wave, say, increases steadily with depth, the amplitude of the motion it produces on emerging at the surface will vary smoothly with distance from the origin; but if the rate of increase of the velocity with depth changes suddenly, the amplitude will also change suddenly. As a rule, observations of the same shock at many stations are not collected, and use was made, instead, of the ratios of the amplitudes of the primary and first reflected waves for different earthquakes; this plan has obvious drawbacks, but it has the advantage that all the observations were made on the same instrument. The method is very sensitive, and its use probably lies mainly in filling in the finer details of the variation of velocity, the times of transmission giving the general outline. A rather sudden decrease of the velocity of the compressional waves, at a depth of about 0.6 of the radius, was inferred from the time-curves by R. D. Oldham in I906. Gutenberg shows that this is confirmed by the smallness of these waves for distances of $110^{\circ}-140^{\circ}$, while their reappearance at minimum deviation is indicated by the large amplitudes found at about $140^{\circ}$. That the central core below this depth is truly fluid is indicated by the absence of distortional waves at greater distances.

An account is given of the "individual P-waves" of A. and S. Mohorovičic--compressional waves from foci within the outer granitic layer, which travel directly from the focus to the surface, whereas the normal $\mathrm{P}$-wave travels down to the basic rocks below and up again. These and explosion waves give valuable information about the surface layer, and the author then discusses the surface waves at length. The estimate of the thickness of the surface layer based on these last is, however, vitiated by a confusion between wave-velocity and group-velocity.

Other chapters deal with the figure of the earth, the internal distribution of density, chemical composition, the cooling of the earth, and the structure of the atmosphere. The age of the earth and tidal friction are not discussed, but the bodily tide is considered at some length. The author has misunderstood the situation with regard to the explanation of the annual variation of latitude, for he suggests that the work of the present reviewer on this subject was an attempt to improve on that of Schweydar, which was already perfect. Actually my work was published in I9I6, Schweydar's in rgig (of course, independently), and in both there remains a considerable discrepancy between theory and observation.

Dr. Gutenberg's book is an important contribution to geophysics, both for its own content and for the aid it gives in the search for other literature.

H. JEFFREYS.

NO. 2933, VOL. II 7 ] 